\title{
Efficient Compute-and-Forward Network Codes Search for Two-Way Relay Channel
}

\author{
Lili Wei, Member, IEEE, and Wen Chen, Senior Member, IEEE
}

\begin{abstract}
We consider the two-way relay channel (TWRC) with compute-and-forward network coding strategy. First a new lemma is proposed as network codes search criteria for TWRC. Then, instead of exhaustive search, we present an efficient network codes search algorithm based on modified Fincke-Pohst method. Numerical results demonstrate the effectiveness and complexity reduction of our proposed lemma and algorithm.
\end{abstract}

Index Terms-Compute-and-forward, two-way relay channel, lattice codes, network coding, cooperative.

\section{INTRODUCTION}

$\mathbf{S}$ INCE the pioneering research work of Ahlswede et al. [1], network coding (NC) has rapidly emerged as a major research area in electrical engineering and computer science. NC is a generalized routing approach that breaks the traditional assumption of simply forwarding data, and allows the intermediate nodes to send out functions of their received packets, by which the multicast capacity can be achieved. Subsequent works [2]-[3] made the important observation that, for multicasting, intermediate nodes can simply send out a linear combination of their received packets. Linear network coding with random coefficients is considered in [4]. Physical layer network coding for wireless system is presented in [5].

Recently, a new network coding strategy of compute-andforward (CPF) [6]-[7] attracts great attention. The main idea is that, a relay will decode a linear combination of the transmitted messages according to the observed channel coefficients. Upon utilizing the algebraic structure of lattice codes [9]-[10], i.e. the integer combination of lattice codewords is still a codeword as well, the intermediate relay node decodes and forwards an integer combination of original messages based on designed network coding vector.

In this work, we consider CPF network coding strategy in the two-way relay channel (TWRC) [11], where two sources exchange information through a relay. First we modify the previous general results in [6]-[8], add the no zero entry constraints and propose a new lemma as network codes search criteria for TWRC. Furthermore, we present in detail an optimal network codes search algorithm for TWRC based on Fincke-Pohst method [12], which returns the same solution as exhaustive search with much lower complexity. The proposed new lemma and algorithm lay a solid foundation for CPF network codes search for TWRC.

Manuscript received March 4, 2012. The associate editor coordinating the review of this letter and approving it for publication was A. Burr.

The authors are with the Department of Electronic Engineering, Shanghai Jiao Tong University, China (e-mail: \{liliwei, wenchen $\} @$ sjtu.edu.cn).W. Chen is also with SKL for ISN, Xidian University, China.

This work is supported by the National 973 Project \#2012CB316106, by NSF China \#60972031 and \#61161130529, by the National 973 Project \#2009CB824904, and by the National Key Laboratory Project \#ISN11-01.

Digital Object Identifier 10.1109/LCOMM.2012.052112.120469

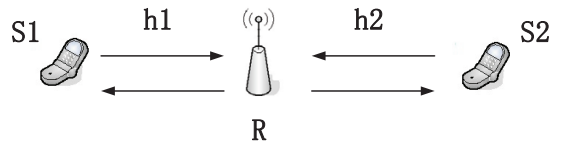

Fig. 1. TWRC model.

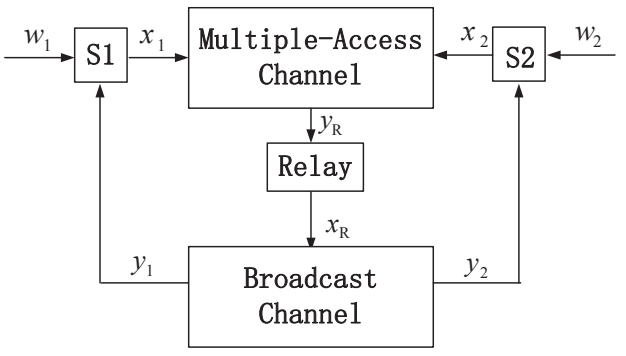

Fig. 2. TWRC diagram.

\section{SySTEM MOdeL}

Consider the classic TWRC with two sources $\mathcal{S}_{1}, \mathcal{S}_{2}$ attempting to exchange information with each other through a relay $\mathcal{R}$ as in Fig. 1. There is no direct link between two sources and each node is equipped with one antenna.

Without loss of generality, in one information codeword transmission, each source has a length- $k$ information vector $\mathbf{w}_{m} \in \mathbb{F}_{p}^{k}, m=1,2$, where $\mathbb{F}_{p}=\{0,1, \cdots, p-1\}$ is a prime size finite field. Each source is equipped with an encoder $\mathcal{E}_{m}: \mathbb{F}_{p}^{k} \rightarrow \mathbb{R}^{n}$ that maps the length- $k$ message $\mathbf{w}_{m}$ into a length- $n$ lattice codeword $\mathbf{x}_{m} \in \mathbb{R}^{n}$. The codeword satisfies the power constraint of $\frac{1}{n}\left\|\mathbf{x}_{m}\right\|^{2} \leq P$.

The information transmission includes two phases. In the first phase, two sources $\mathcal{S}_{1}, \mathcal{S}_{2}$ transmit simultaneously to relay $\mathcal{R}$, which can be modeled by a multiple-access channel with inputs $\mathbf{x}_{1}, \mathbf{x}_{2}$ and output $\mathbf{y}_{R}$. In the second phase, relay $\mathcal{R}$ broadcast to $\mathcal{S}_{1}$ and $\mathcal{S}_{2}$, which can be modeled by a broadcast channel with input $\mathbf{x}_{R}$ and outputs $\mathbf{y}_{1}$ and $\mathbf{y}_{2}$. The transmission diagram of TWRC is shown in Fig. 2.

At the end of first phase, relay $\mathcal{R}$ will receive

$$
\mathbf{y}_{R}=h_{1} \mathbf{x}_{1}+h_{2} \mathbf{x}_{2}+\mathbf{z}_{R},
$$

where $h_{1}, h_{2} \in \mathbb{R}$ are real valued fading channel coefficient from $\mathcal{S}_{1}$ and $\mathcal{S}_{1}$ to relay $\mathcal{R}$ respectively and $\mathbf{z}_{R} \in \mathbb{R}^{n}$ is additive Gaussian noise. All channel coefficients are generated i.i.d. according to a normal distribution $\mathcal{N}(0,1)$.

In the framework of CPF [6], the property that any integer combination of lattice codewords is again a lattice codeword is exploited. After receiving the noisy vector $\mathbf{y}_{R}$, relay $\mathcal{R}$ will select a scalar $\beta \in \mathbb{R}$ and an integer network coding vector $\mathbf{a}=\left[a_{1}, a_{2}\right]^{T} \in \mathbb{Z}^{2}$, and attempts to decode 


$$
\begin{aligned}
\mathbf{x}_{R}=a_{1} \mathbf{x}_{1} & +a_{2} \mathbf{x}_{2} \text { from } \\
\beta \mathbf{y}_{R} & =\beta h_{1} \mathbf{x}_{1}+\beta h_{2} \mathbf{x}_{2}+\beta \mathbf{z}_{R} \\
& =a_{1} \mathbf{x}_{l}+a_{2} \mathbf{x}_{2}+\underbrace{\sum_{m=1}^{2}\left(\beta h_{m}-a_{m}\right) \mathbf{x}_{m}+\beta \mathbf{z}_{R}}_{\text {Effective Noise }} .
\end{aligned}
$$

At the end of second phase, $\mathcal{S}_{1}, \mathcal{S}_{2}$ will receive respectively

$$
\begin{aligned}
& \mathbf{y}_{1}=h_{1}\left(a_{1} \mathbf{x}_{1}+a_{2} \mathbf{x}_{2}\right)+\mathbf{z}_{1} \\
& \mathbf{y}_{2}=h_{2}\left(a_{1} \mathbf{x}_{1}+a_{2} \mathbf{x}_{2}\right)+\mathbf{z}_{2} .
\end{aligned}
$$

Then, each source can subtract its own signal and attempt to decode for the other source.

At the relay, we are interested in the rate of $a_{1} \mathbf{x}_{1}+a_{2} \mathbf{x}_{2}$ as a whole and capture the performance by what refer to as the computation rate, namely, the number of bits of the integer linear function successfully recovered per channel use. The role of $\beta$ can be thought as trying to move the channel coefficients toward integers [7]. We conclude the results regarding CPF network coding in [6]-[8] in the following theorems. Denote channel vector $\mathbf{h}=\left[h_{1}, h_{2}\right]^{T}$ and $\log ^{+}(x) \triangleq \max (\log (x), 0)$.

Theorem 2.1: For real-valued AWGN network with channel vector $\mathbf{h}$ and network coding vector $\mathbf{a}$, the following computation rate is achievable

$$
\mathscr{R}(\mathbf{a})=\max _{\beta \in \mathbb{R}} \frac{1}{2} \log ^{+}\left(\frac{P}{\beta^{2}+P\|\beta \mathbf{h}-\mathbf{a}\|^{2}}\right) .
$$

Theorem 2.2: The computation rate given in Theorem 2.1 is uniquely maximized by choosing $\beta$ to be the MMSE coefficient

$$
\beta_{M M S E}=\frac{P \mathbf{h}^{T} \mathbf{a}}{1+P\|\mathbf{h}\|^{2}},
$$

which results in

$$
\mathscr{R}(\mathbf{a})=\frac{1}{2} \log ^{+}\left(\|\mathbf{a}\|^{2}-\frac{P\left(\mathbf{h}^{T} \mathbf{a}\right)^{2}}{1+P\|\mathbf{h}\|^{2}}\right)^{-1} .
$$

Theorem 2.3: For a given channel vector $\mathbf{h}, \mathscr{R}(\mathbf{a})$ is maximized by choosing the integer network coding vector a as

$$
\mathbf{a}=\arg \min _{\mathbf{a} \in \mathbb{Z}^{2}, \mathbf{a} \neq \mathbf{0}}\left(\mathbf{a}^{T} \mathbf{G a}\right)
$$

with constraint $\|\mathbf{a}\|^{2} \leq 1+P\|\mathbf{h}\|^{2}$ and $\mathbf{G} \triangleq \mathbf{I}-\frac{P\left(\mathbf{h} \mathbf{h}^{T}\right)}{1+P\|\mathbf{h}\|^{2}}$.

\section{OPTIMAL NETWORK CODES SEARCH FOR TWRC}

\section{A. Formulation}

Theorems 2.1-2.3 only give the general criteria to search the optimal network coding integer vector a at relay $\mathcal{R}$ and do not take consideration of the specific system constraints. For TWRC, in order to let each source receive signals from the other one through relay $\mathcal{R}$, there should be no zero entry in network coding vector $\mathbf{a}=\left[a_{1}, a_{2}\right]^{T}$, i.e. $a_{1} \neq 0$ and $a_{2} \neq 0$ must be satisfied at the same time. In other words, network coding vector in form of $\left[a_{1}, 0\right]^{T}$ or $\left[0, a_{2}\right]^{T}$ will fail the information transmission for TWRC.

Hence, we modify theorem 2.3 and propose a new network coding vector search criteria for TWRC as follows.
Lemma 3.1: In TWRC, for a given channel vector $\mathbf{h}, \mathscr{R}(\mathbf{a})$ is maximized by choosing the integer network coding vector a as

$$
\begin{aligned}
& \qquad \mathbf{a}=\arg \min _{\mathbf{a} \in \mathbb{Z}^{2}, a_{1} \neq 0, a_{2} \neq 0}\left(\mathbf{a}^{T} \mathbf{G a}\right) \\
& \text { with constraint }\|\mathbf{a}\|^{2} \leq 1+P\|\mathbf{h}\|^{2} \text { and } \mathbf{G} \triangleq \mathbf{I}-\frac{P\left(\mathbf{h} \mathbf{h}^{T}\right)}{1+P\|\mathbf{h}\|^{2}} \text {. }
\end{aligned}
$$

A direct approach to this optimization problem in Lemma 3.1 will be exhaustive search among all integer vectors satisfying $\|\mathbf{a}\|^{2} \leq 1+P\|\mathbf{h}\|^{2}$. However, as the power constraint $P$ gets larger, the number of candidate vectors increases dramatically. In this work, we will propose an algorithm based on modified Finche-Pohst (FP) method, to searching within a much smaller candidate set with the optimal solution included.

Operate Cholesky's factorization of matrix $\mathbf{G}, \mathbf{G}=\mathbf{U}^{T} \mathbf{U}$, where $\mathbf{U}$ is an upper triangular matrix. Then, the optimization of (9) becomes

$$
\mathbf{a}=\arg \min _{\mathbf{a} \in \mathbb{Z}^{2}, a_{1} \neq 0, a_{2} \neq 0}\|\mathbf{U} \mathbf{a}\|_{F}^{2} .
$$

The original FP method [12] searches through the integer points a in Euclidean space, which make the corresponding vectors $\mathbf{z} \triangleq \mathbf{U a}$ inside a sphere of given radius $\sqrt{C}$ centered at the origin point, i.e. $\|\mathbf{U} \mathbf{a}\|_{F}^{2}=\|\mathbf{z}\|_{F}^{2} \leq C$. This guarantees that only the points that make the corresponding vectors z within the square distance $C$ from the origin point are considered in the metric minimization.

Compared with the original FP algorithm, we have two main modifications: $(i)$ We add two constraints: the no zero entry constraint and $\|\mathbf{a}\|^{2} \leq 1+P\|\mathbf{h}\|^{2}$ constraint to search for the optimal network coding vector in TWRC. (ii) According to the binary vector obtained by applying the direct sign operator on the real minimum-eigenvalue eigenvector of $\mathbf{G}$, denoted as $\mathbf{a}_{\text {quant }}$, we can have a very proper square distance setting as

$$
C=\mathbf{a}_{\text {quant }}^{T} \mathbf{G} \mathbf{a}_{\text {quant }},
$$

such that the searching sphere radius is big enough to have at least one searching point fall inside, while in the meantime small enough to have only a few within. We calculate the $\mathbf{a}^{T} \mathbf{G a}$ metric for every candidate vector that satisfies $\|\mathbf{U a}\|_{F}^{2} \leq C$, such that the optimal network coding vector with minimum $\mathbf{a}^{T} \mathbf{G a}$ metric (maximizing the computation rate for relay $\mathcal{R}$ equivalently) is obtained from the modified FP algorithm directly.

Since the radius is fixed for our modified FP algorithm, the complexity uncertainty due to the radius update, which means that the radius need to be expanded if no points found in the sphere and the radius need to be reduced if too many points found within as shown in the literature of sphere decoding, is not a question in this optimization.

\section{B. Searching Algorithm Derivation}

Let $u_{i j}, i, j=1,2$, be entries of matrix $\mathbf{U}$. Then, the searching vector $\mathbf{a}$ that makes $\mathbf{a}^{T} \mathbf{G a} \leq C$ can be expressed as

$$
\begin{aligned}
& \mathbf{a}^{T} \mathbf{G a}=\|\mathbf{U} \mathbf{a}\|_{F}^{2}=\quad\left(u_{11} a_{1}+u_{12} a_{2}\right)^{2}+\left(u_{22} a_{2}\right)^{2} \\
& =g_{11}\left(a_{1}+g_{12} a_{2}\right)^{2}+g_{22} a_{2}^{2} \leq C
\end{aligned}
$$


where $g_{i i}=u_{i i}^{2}$ for $i=1,2$ and $g_{12}=u_{12} / u_{11}$. To satisfy (12), it is equivalent to consider,

$$
\left\{\begin{array}{l}
g_{22} a_{2}^{2} \leq C \\
g_{11}\left(a_{1}+g_{12} a_{2}\right)^{2}+g_{22} a_{2}^{2} \leq C
\end{array}\right.
$$

We begin with evaluation of the entry $a_{2}$. Set $\Delta_{2}=0$, $C_{2}=C$. Referring to (13), we get

$$
L B_{2} \leq a_{2} \leq U B_{2},
$$

and

$$
U B_{2}=\left\lfloor\sqrt{\frac{C_{2}}{g_{22}}}-\Delta_{2}\right\rfloor, L B_{2}=\left\lceil-\sqrt{\frac{C_{2}}{g_{22}}}-\Delta_{2}\right\rceil .
$$

where $\lceil x\rceil$ is the smallest integer no less than $x$ and $\lfloor x\rfloor$ is the greatest integer no bigger than $x$. The candidate for $a_{2}$ is chosen as an integer inside its bound requirement (14)-(15) and excludes zero.

To evaluate $a_{1}$, set $\Delta_{1}=g_{12} a_{2}, C_{1}=C-g_{22} a_{2}^{2}$. Referring to (13) we will have

$$
L B_{1} \leq a_{1} \leq U B_{1},
$$

and

$$
U B_{1}=\left\lfloor\sqrt{\frac{C_{1}}{g_{11}}}-\Delta_{1}\right\rfloor, L B_{1}=\left\lceil-\sqrt{\frac{C_{1}}{g_{11}}}-\Delta_{1}\right\rceil .
$$

Then $a_{1}$ is chosen as an integer inside its bound requirement (16)-(17) and excludes zero.

The entries $a_{2}, a_{1}$ are chosen as follows: for a chosen $a_{2}$ that satisfies its bound requirement (14)-(15) and $a_{2} \neq 0$, we can choose $a_{1}$ satisfying its bounds requirements (16)-(17) and $a_{1} \neq 0$. If such $a_{1}$ does not exist, we go back and choose other $a_{2}$. Then search for $a_{1}$ that meets its bounds requirement for this new $a_{2}$ and $a_{1} \neq 0$. When a set of $a_{2}, a_{1}$ is chosen, we test the $\|\mathbf{a}\|^{2} \leq 1+P\|\mathbf{h}\|^{2}$ constraint. If satisfied, one candidate network coding vector $\mathbf{a}=\left[a_{1}, a_{2}\right]^{T}$ is obtained. We choose the one among all network coding candidate vectors that gives the smallest $\mathbf{a}^{T} \mathbf{G a}$ metric, which equivalently maximizes the computation rate at relay $\mathcal{R}$.

Note that this searching procedure will return all candidates that satisfy $\mathbf{a}^{T} \mathbf{G a} \leq C$, and gives the one with minimum value. There is at least one candidate vector $\mathbf{a}_{\text {quant }}$ such that its entries satisfy all the bounds requirements, since that is how we set the radius value in (11). On the other hand, the exhaustive search result $\mathbf{a}_{\text {exhaustive }}$ that returns the minimum metric will also fall inside the search bounds, since

$$
\mathbf{a}_{\text {exhaustive }}{ }^{T} \mathbf{G} \mathbf{a}_{\text {exhaustive }} \leq \mathbf{a}_{\text {quant }}{ }^{T} \mathbf{G} \mathbf{a}_{\text {quant }}=C \text {. }
$$

Hence, we are guaranteed to find the optimal exhaustive search result by the proposed algorithm. Simulation results in Section IV also demonstrate this optimality.

\section{Optimal Network Codes Search Algorithm for TWRC}

We summarize our proposed algorithm to search the optimal network coding vector for TWRC as follows.

\section{Optimal Network Codes Search Algorithm for TWRC}

Input: Channel coefficient vector $\mathbf{h}$.
Output: The optimal network coding vector $\mathbf{a}_{\min }$ for TWRC. Step 1: Based on the channel coefficient vector $\mathbf{h}$, construct matrix $\mathbf{G}$ as $\mathbf{G}=\mathbf{I}-\frac{P\left(\mathbf{h h}^{T}\right)}{1+P\|\mathbf{h}\|^{2}}$.

Step 2: Calculate the binary quantized vector obtained by applying the direct sign operator of the real minimum-eigenvalue eigenvector of $\mathbf{G}$, denoted as $\mathbf{a}_{\text {quant }}$, and set $C$ as

$$
C=\mathbf{a}_{\text {quant }}^{T} \mathbf{G} \mathbf{a}_{\text {quant }} .
$$

Step 3: Operate Cholesky's factorization of matrix $\mathbf{G}, \mathbf{G}=$ $\overline{\mathbf{U}^{T} \mathbf{U}}$. Let $u_{i j}, i, j=1,2$ denote the entries of matrix $\mathbf{U}$. Set $g_{i i}=u_{i i}^{2}$ for $i=1,2$ and $g_{12}=u_{12} / u_{11}$.

Step 4: Search the candidate vector $\mathbf{a}=\left[a_{1}, a_{2}\right]^{T}$ according to the following procedure.

(i) Initialize $\Delta_{2}=0, C_{2}=C$, metric $=C, \mathbf{a}_{\text {min }}=\mathbf{a}_{\text {quant }}$ and $k=2$.

(ii) Set the upper bound $U B_{k}$ and the lower bound $L B_{k}$ as follows

$$
U B_{k}=\left\lfloor\sqrt{\frac{C_{k}}{g_{k k}}}-\Delta_{k}\right\rfloor, \quad L B_{k}=\left\lceil-\sqrt{\frac{C_{k}}{g_{k k}}}-\Delta_{k}\right\rceil
$$

and $a_{k}=L B_{k}-1$.

(iii) Set $a_{k}=a_{k}+1$. If $a_{k}=0$, move to $a_{k}=1$. For $a_{k} \leq U B_{k}$, go to (v); else go to (iv).

(iv) If $k=2$, terminate and output the searching result $\mathbf{a}_{\min }$; else set $k=k+1$ and go to (iii).

(v) For $k=1$, go to (vi); else set $k=k-1$, and

$$
\Delta_{1}=g_{12} a_{2}, \quad C_{1}=C-g_{22} a_{2}^{2},
$$

then go to (ii).

(vi) Test for $\|\mathbf{a}\|^{2} \leq 1+P\|\mathbf{h}\|^{2}$ constraint to get a candidate vector $\mathbf{a}$. If $\mathbf{a}^{T} \mathbf{G a} \leq$ metric, update $\mathbf{a}_{\min }=\mathbf{a}$ and metric $=\mathbf{a}^{T} \mathbf{G a}$. Go to (iii).

\section{EXPERIMENTAL STUDIES}

We present experimental studies to demonstrate the effectiveness of our proposed lemma and algorithm. First, with the average of 10000 randomly generated channel realizations, we show in Fig. 3 that if network coding vector is searched based on general criteria of theorem 2.3 without our lemma, the probability that the resulting vector will have at least one zero entry and fails the TWRC system. It can be observed that this issue is actually very severe. For example, with $P \leq 15 \mathrm{~dB}$, the probability of zero entry is always beyond $1 / 2$.

In Fig. 4. we compare the average rate of several strategies under TWRC model. (i) Relay $\mathcal{R}$ decode and transmit $\mathrm{x}_{1}+\mathbf{x}_{2}$ to both sources, which we denote as "DF-NC". This strategy can been seen as static network coding as it does not consider the channel variations. (ii) Relay $\mathcal{R}$ decode a linear integer combination of both sources, while the network coding vector are set by simply rounding channel vector $\mathbf{h}$ directly, denoted as "HNC". (iii) The general CPF scheme while the network coding vector is optimized without our proposed lemma. We denote as "CPF without zero entry constraints". (iv) The CPF scheme under our proposed lemma and algorithm for TWRC, denoted as "CPF with proposed algorithm". (v) The CPF scheme under our proposed lemma 
TABLE I

COMPLEXITY COMPARISON: AVERAGE NUMBER OF CANDIDATE VECTORS

\begin{tabular}{|c|c|c|c|c|c|c|c|}
\hline$P$ & $0 \mathrm{~dB}$ & $5 \mathrm{~dB}$ & $10 \mathrm{~dB}$ & $15 \mathrm{~dB}$ & $20 \mathrm{~dB}$ & $25 \mathrm{~dB}$ & $30 \mathrm{~dB}$ \\
\hline Proposed & 1.1970 & 1.9388 & 3.1606 & 5.5110 & 10.3098 & 20.0808 & 38.6586 \\
\hline Exhaustive & 1.8188 & 7.1324 & 25.7178 & 86.7106 & 287.6744 & 957.2552 & 3073.03 \\
\hline
\end{tabular}

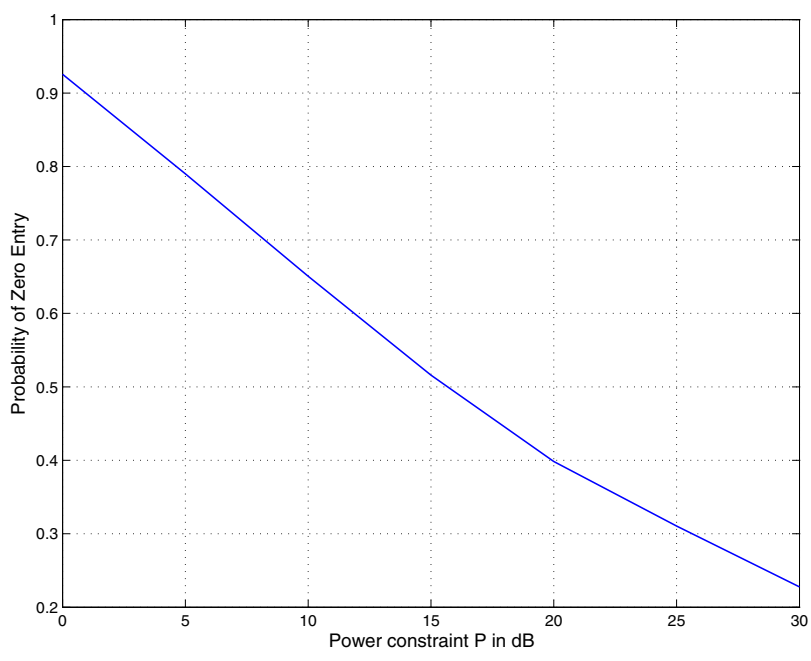

Fig. 3. Probability of zero entry in TWRC.

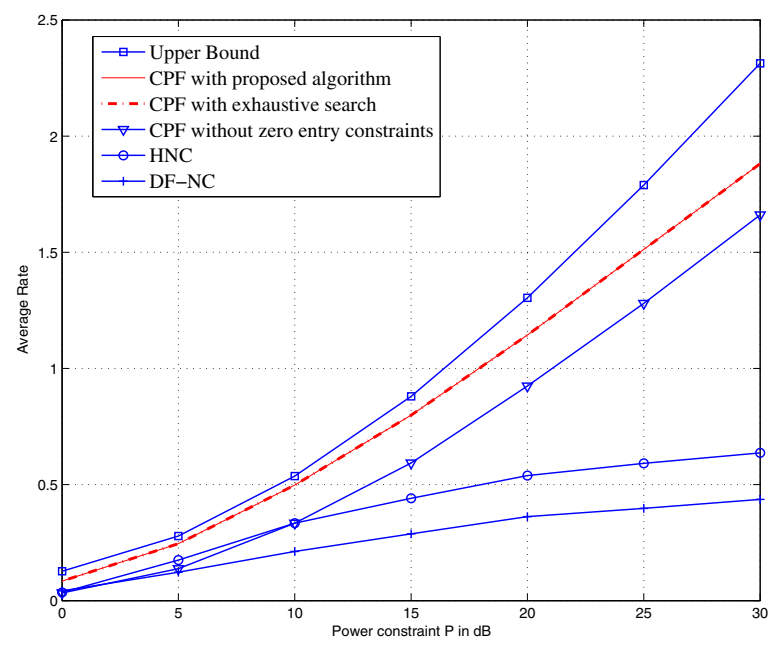

Fig. 4. Average rate comparisons for TWRC.

and exhaustive search, denoted as "CPF with exhaustive search". (vi) The "Upper Bound" on the computation rate, $\mathscr{R}_{\text {Upper }}=\min _{m=1,2} \frac{1}{2} \log \left(1+\left|h_{m}\right|^{2} P\right)$ [6].

As show in Fig. 4, the performance differences are apparent. "DF-NC" gives very poor result compared with other strategies since the network coding vector is not adaptive to the changing channel. "HNC" strategy somehow consider network coding adaptively, but the network coding vector is chosen in a simplified way and not optimal. "CPF with proposed algorithm" performs superior to other practical strategies and actually overlaps with the "CPF with exhaustive search" curve. It further verifies the optimality of our proposed algorithm which produces the same solution as exhaustive search.

Finally, to demonstrate the complexity reduction of proposed algorithm with exhaustive search, in Table 1 we show, over 10000 randomly generated channel realizations, the statistical average number of network coding candidate vectors need to be searched to find the optimum solution. We can see that the candidate set is reduced significantly by our proposed algorithm hence lower the complexity dramatically. For example, when $P=15 d B$, there are in average 86.7106 integer vectors need to considered for $\mathbf{a}^{T} \mathbf{G a}$ calculation to get the optimal network coding vector for exhaustive search, while with our proposed algorithm, we only need to consider in average 5.5110 integer vectors. Hence, our proposed algorithm has the same optimal solution as exhaustive search with much lower complexity.

\section{REFERENCES}

[1] R. Ahlswede, N. Cai, S. R. Li, and R. W. Yeung, "Network information flow," IEEE Trans. Inf. Theory, vol. 46, no. 4, pp. 1204-1216, July 2000.

[2] S. R. Li, R. Ahlswede, and N. Cai, "Linear network coding," IEEE Trans. Inf. Theory, vol. 49, no. 2, pp. 371-381, Feb. 2003.

[3] R. Koetter and M. Medard, "An algebraic approach to network coding," IEEE/ACM Trans. Networking, vol. 11, no. 5, pp. 782-795, Oct. 2003.

[4] T. Ho, R. Koetter, M. Medard, D. R. Karger, M. Effros, J. Shi, and B. Leong, "A random linear network coding approach to multicast," IEEE Trans. Inf. Theory, vol. 52, no. 10, pp. 4413-4430, Oct. 2006.

[5] S. Zhang, S. Liew, and P. P. Lam, "Physical-layer network coding," in Proc. 2006 IEEE MobiComm, pp. 358-365.

[6] B. Nazer and M. Gastpar, "Compute-and-forward: harnessing interference through structured codes," IEEE Trans. Inf. Theory, vol. 57, no. 10, Oct. 2011.

[7] B. Nazer and M. Gastpar, "Reliable physical layer network coding," Proc. IEEE, vol. 99, no. 3, pp. 438-460, Mar. 2011.

[8] A. Osmane and J. C. Belfiore, "The compute-and-forward protocol: implementation and practical aspects," IEEE Commun. Lett., submitted. Available online: http://arxiv.org/abs/1107.0300.

[9] R. Zamir, "Lattices are everywhere," in Proc. 2009 Workshop on Info. Theory and its Applications.

[10] U. Erez and R. Zamir, "Achieving (1/2) $\log (1+\mathrm{SNR})$ on the AWGN channel with lattice encoding and decoding," IEEE Trans. Inf. Theory, vol. 50, no. 10, pp. 2293-2314, Oct. 2004.

[11] Y. Wu, P. A. Chou, and S. Kung, "Information exchange in wireless networks with network coding and physical-layer broadcast," Microsoft Research, Redmond, WA, Tech. Rep. MSR-TR-2004-78, Aug. 2004.

[12] U. Fincke and M. Pohst, "Improved methods for calculating vectors of short length in a lattice, including a complexity analysis," Math. Comput., vol. 44, pp. 463-471, Apr. 1985.

[13] E. Viterbo and J. Boutros, "A universal lattice code decoder for fading channels," IEEE Trans. Inf. Theory, vol. 45, no. 5, pp. 1635-1642, July 1999.

[14] O. Damen, A. Chkeif, and J. C. Belfiore, "Lattice code decoder for space-time codes," IEEE Commun. Lett., vol. 4, no. 5, pp. 161-163, May 2000. 